\title{
Research and Analysis for Planetary Defense In Situ Spacecraft Missions to Near-Earth Objects
}

\author{
Brent W. Barbee \\ 301-286-1837 (Office) / 301-448-5681 (Cell) \\ NASA/Goddard Space Flight Center (GSFC) \\ brent.w.barbee@nasa.gov / bbarbee@umd.edu
}

Paul A. Abell (NASA Johnson Space Center (JSC))

Daniel R. Adamo (Independent Astrodynamics Consultant)

Justin Atchison (Johns Hopkins University Applied Physics Laboratory (JHUAPL))

Olivier Barnouin (Johns Hopkins University Applied Physics Laboratory (JHUAPL))

Joshua Cahill (Johns Hopkins University Applied Physics Laboratory (JHUAPL))

Paul Chodas (CalTech/Jet Propulsion Laboratory (JPL))

Terik Daly (Johns Hopkins University Applied Physics Laboratory (JHUAPL))

Amanda Davis (Johns Hopkins University Applied Physics Laboratory (JHUAPL))

Jessie Dotson (NASA/Ames Research Center (ARC))

Dan Mazanek (NASA/Langley Research Center (LARC))

Ryan Park (CalTech/Jet Propulsion Laboratory (JPL))

Clemens Rumpf (STC at NASA/Ames Research Center (ARC))

Thomas S. Sotirelis (Johns Hopkins University Applied Physics Laboratory (JHUAPL))

Lorien Wheeler (NASA/Ames Research Center (ARC)) 


\section{Introduction}

This paper summarizes important areas of study to support spacecraft mission design for in situ planetary defense missions to near-Earth objects (NEOs), including missions for reconnaissance (characterization, monitoring mitigation attempts, etc.) and mitigation (deflection/disruption). The topics described here include: planetary defense mission design and relevant properties of an Earth-impacting NEO; reconnaissance/characterization of NEOs for planetary defense purposes; uncertainties in NEO properties, associated modeling uncertainties, and methods for addressing those uncertainties in the mission design process; launch vehicle infrastructure and mission architectures for planetary defense campaigns; and the need for research addressing unique mission design challenges posed by long-period/hyperbolic comets. These planetary defense research and analysis topics map to actions in the National Near-Earth Object Preparedness Strategy and Action Plan (NSTC, 2018), which specifies actions US government agencies should take to improve our ability to respond to a hazardous NEO. Research and analysis activities must enable fundamental advances in each of these areas during the next decade.

\section{NEO Characterization Priorities for Planetary Defense}

When an NEO is identified as a potential Earth impactor, a wide range of parameters available about the NEO will be used to compute mission options for reconnoitering the NEO and mitigating the threat it poses. At the same time, computer models will be used to estimate the range of possible impact effects if the NEO were to strike Earth. The NEO's orbital state and physical properties, in particular, are crucial inputs to the estimation of impact risk (Mathias et al., 2017) and to predict mitigation mission outcome (Rumpf et al., 2020; Eggl et al., 2015). The analytical results of these planetary defense calculations must be statistical and include uncertainties in the available information for the NEO.

Interagency efforts between NASA and the Department of Energy (DOE) National Nuclear Security Administration (NNSA) laboratories have produced a notionally prioritized list of NEO properties to be characterized during a planetary defense scenario (Barbee, 2020). However, how such priorities may vary across planetary defense scenarios must be assessed, and the accuracy requirements for NEO properties knowledge must be determined.

NEO characterization data return is likely to differ between reconnaissance missions performing a high-speed NEO flyby and missions that rendezvous with the NEO. While rendezvous missions will always provide the most precise, accurate, and complete information on an NEO, a flyby provides several potential advantages. Launch opportunities are usually more available for a flyby than a rendezvous, and a flyby mission usually requires less propellant than a rendezvous. Therefore, it is possible for flyby missions to be more suited to rapid-response than rendezvous missions. However, the ability of high-speed spacecraft flybys to gather sufficient data on an NEO for planetary defense purposes is uncertain, and these issues require further research and analysis.

Some NEO properties to be measured for planetary defense purposes depend on or are related to the NEO's interior structure. However, we have yet to directly measure the interior structure of an NEO. Interior structure (e.g., rubble pile vs. fractured shard vs. monolith) will influence the outcome of deflection and disruption attempts (Asphaug et al., 1998; Huebner and Greenberg, 2002; Michel, 2013; Dearborn et al., 2020) and the assessment of potential atmospheric breakup, damage, and risk should the object strike Earth (Wheeler et al., 2018). Assessing the range of NEO 
interior structures (via radar tomography or seismic sounding) would provide valuable information for mission planning and impact risk assessment.

Related to this is the risk of unintentional NEO fragmentation during a deflection attempt. The 2019 Planetary Defense Conference report (IAA Planetary Defense Conference, 2019) noted, "Uncertainty in how much applied deflection "Delta-V" (change-in-velocity) an NEO can absorb without accidental fragmentation continues to cause difficulties in designing and sizing NEO deflection missions. When is the imparted Delta-V too high? Will dividing the Delta-V into smaller applications via multiple spacecraft avoid accidental NEO fragmentation? How many spacecraft/launches are needed? These considerations can dramatically affect the required size, cost, complexity, and development timeline for mitigation missions and need to be understood well enough for effective planning and implementation of missions in a real scenario."

Finally, the community lacks adequate uncertainty quantification in the outcomes of nuclear deflection/disruption attempts. Modeling in support of NASA's upcoming Double Asteroid Redirection Test (DART) has improved our understanding of asteroid properties that have the most leverage over the outcome of deflection via a kinetic impactor (e.g., Bruck Syal et al., 2016; Dearborn et al., 2020; Jutzi \& Michel, 2014; Lomov et al., 2013; Michel, 2013; Rainey et al., 2020; Stickle et al., 2020). A similar level of information is not available in the open literature for nuclear deflection attempts. The 2019 Planetary Defense Conference report (IAA Planetary Defense Conference, 2019) noted, "Uncertainties associated with the deflection imparted to a NEO via a nuclear device need to be studied (similar to the way that the so-called "momentum enhancement factor," or "beta," is studied for kinetic impactors)." These results should be reported in the open literature, to the extent possible given the sensitivity of nuclear-related information.

\section{Development of Risk-Informed Mission Design Processes for Planetary Defense}

Mission designers and decision makers will face a deficiency in the information available for assessing and mitigating a threatening NEO. This information gap arises because of the long leadtime required to build a mitigation mission and to have it arrive at the NEO years before impact to accomplish an effective mitigation (IAA Planetary Defense Conference, 2019). The current approach in NEO threat response exercises is to use a point design solution, which can have substantial residual risk and unintended consequences due to not accounting for all uncertainties in the NEO's orbit and physical properties. Sampling the full uncertainty ranges is required to facilitate effective decision-making and mission design under realistic levels of knowledge. The overarching goal is integrating risk estimation, with uncertainties, into mitigation mission design.

The uncertain properties of the NEO can be represented as statistical probability density functions (PDFs). A Monte Carlo approach samples the PDFs for each property to generate millions of property sets. Fast-running, analytical models enable Earth impact simulations with these sample sets (Silber et al., 2018) and provide statistical risk results for the possible Earth impact scenarios (Mathias et al., 2017). These models need to be integrated with mission design tools to enable seamless, risk-informed mitigation mission optimization. It is also possible to study the expected outcome of mitigation missions using analytical models that estimate the deflection impulse based on NEO and mission properties (Dearborn et al., 2020; Housen \& Holsapple, 2011). Results inform the mitigation mission design process by providing feedback on adequate sizing of the mitigation mission in order to reduce the impact risk to manageable levels while taking into account the full 
uncertainty range in asteroid properties and mitigation mission outcome (Rumpf et al., 2020). This also includes investigation of asteroid disruption consequences. NEO disruption could intentionally break up a NEO into smaller pieces to reduce the threat to Earth, or it could be an unintended consequence of a deflection mission that could lead to fragments hitting the Earth in new locations. Probabilistic risk analysis of disruption can prompt design changes accordingly.

This approach is also relevant for simulating impact effects and deflection mission outcomes based on physical property uncertainties, and can help prioritize NEO characterization or reconnaissance efforts. It can identify unintended mitigation outcomes including moving the NEO's impact location to a new, previously unthreatened region on the Earth. By estimating the range of deflection mission outcomes, these results can inform the socio-political discussions needed to conduct a mitigation mission in a responsible manner. This approach can also be extended to study the guidance laws that direct the deflection force applied to the NEO over time to steer the projected Earth impact point along a low-risk corridor on the surface of the Earth (e.g., avoiding population centers) until full mitigation is achieved (Rumpf, Asteroid Impact Risk, 2016). This would reduce the probability of unintentionally moving the impact point to a new high-risk impact location during the mitigation attempt.

\section{The Planetary Defense Spacecraft Mission Design Parameter Space}

Planetary defense missions pose challenges for a spacecraft trajectory design. The target NEO's orbit may be eccentric and/or highly inclined to the ecliptic plane, which limits launch options and/or imposes high spacecraft propellant mass requirements for post-launch NEO intercept or rendezvous maneuvers. The date of arrival is critical, so time-of-flight is an important variable that can be at odds with the capabilities of solar-electric-propulsion or multiple gravity assist trajectories. For kinetic impactors, the arrival geometry contributes to the NEO deflection outcome but is highly constrained by orbit mechanics. Terminal guidance and impact mechanics impose constraints on the arrival speed and solar illumination from the spacecraft's point of view. Additional research and analyses are needed to improve our understanding of the acceptable ranges for mission design parameters, with the goals of defining best practices for planetary defense mission design assumptions/constraints and producing credible, realistic mission design solutions.

We recommend that a study be conducted to better characterize what combinations of approach velocities, solar phase angles, and NEO sizes are feasible for various mitigation mission concepts, similar to (Hernandez, 2013). These constraints, which should be improved by future research, can be computed using the NEO's position vector and spacecraft's velocity vector at the time of their encounter. An upper bound on the approach velocity magnitude should also be accounted for to ensure that ground or autonomous guidance is possible. (Bhaskaran et al. 2014) simulated encounters with successful guidance at velocities as high as $20 \mathrm{~km} / \mathrm{s}$ for large-enough objects. Similarly, a lower bound on the duration between on-board NEO detection and impact should be accounted for (Sarli, et al. 2017). Additionally, spacecraft sensors must have sufficient sensitivity to detect NEOs down to certain limiting apparent brightness some time before NEO encounter.

\section{Launch Vehicle Infrastructure \& Payload Architecture for Planetary Defense Campaigns}

Planetary defense missions may need to launch with very short warning, which would be challenging with existing infrastructure. The concept of maintaining an intentional surplus inventory of flight hardware to address unforeseeable threats or opportunities was implemented in 
support of the Skylab (Skylab Rescue, 2020) and Space Shuttle (STS-3xx, 2020) programs. These "launch-on-need" (LON) efforts had minimal cost impact because they simply ensured that hardware — which would be launched anyway_-was ready a few months earlier than necessary.

A similar concept could apply to planetary defense missions. However, the orbit in which an Earth impact threat resides is unknown when LON hardware is procured. Delivery to threat orbits could span a broad range of performance requirements. Also, launch cadence is irregular and likely on the order of decades, due to statistical Earth impact rate (Soderman/NLSI Staff, 2013).

To address the impact threat's unknown orbit, a modular LON payload design would be essential. For example, a propulsion module capable of rendezvous with more-accessible threats could be swapped with a detachable impactor module if only a flyby of the NEO is possible. In this manner, a given launch vehicle could be used for reconnaissance or mitigation over a range of threats. Launch azimuths required to access a threat are unknown at LON purchase, so a launch vehicle capable of departing multiple sites such as Vandenburg and Cape Canaveral would be desirable.

The overall architecture of planetary defense mission campaigns also needs to be assessed. Going beyond the LON concept, should entire planetary defense spacecraft (for reconnaissance, mitigation, or both) be completely or partially pre-built and stored to reduce response time? Should we have separate spacecraft for reconnaissance and mitigation, or include capabilities to perform both functions during the same mission? What would become of the shelved spacecraft (or modular components thereof) if they are not needed for planetary defense prior to their expiration dates? Perhaps those spacecraft/components would be sold for use by science missions (either opportunistic or planned in advance), and the funds from those sales would be used to procure replacement spacecraft/components for the next round of storage. The usage of custom-built sensors, instruments, and other components versus commercial-off-the-shelf hardware must also be considered. There is a broad parameter space to be assessed for architecture options.

Similarly, if LON vehicles were procured in advance, an infrequent launch cadence would likely require LON hardware be used for other purposes before its shelf life expired. Ideally, LON hardware resales could leave planetary defense agencies with little more than storage costs to absorb over many yearly budget cycles, or perhaps vehicles could be constantly cycled through a reserve set to avoid the "shelf life" complication altogether. If resale is required, the process could be institutionalized with a rideshare program similar to those already in existence (Caffrey, 2019). In these manners, the LON cadence could be decoupled from the relatively low frequency of credible planetary defense threats and instead associated with the more constant demand for space launch opportunities. If launch vehicle storage for LON proves to be practical, questions remain about how quickly a stored launch vehicle could be made ready to launch a spacecraft. Launch preparations generally include trajectory targeting, analyses, software validation, and other timeintensive preparatory tasks, which typically require at least 6-8 months. Future work should examine how to reduce launch preparation time for planetary defense purposes.

\section{Planetary Defense Mission Performance Needs for Challenging Incoming Comet Scenarios}

Most planetary defense mission design studies to date have focused on scenarios involving hazardous near-Earth asteroids (NEAs) rather than comets, as the probability of Earth impact by a long-period comet is far less than the probability of Earth impact by an NEA. However, comets- 
particularly long-period, (near-)parabolic, or hyperbolic comets - typically pose more challenging scenarios than even NEAs on rather extreme orbits (e.g., orbits with relatively large semi-major axes, eccentricities, and/or inclinations) due to intrinsically shorter warning times and higher performance requirements for spacecraft that might be deployed to reconnoiter or attempt to deflect/disrupt the comet. Additionally, comets are likely to strike the Earth at much higher velocities than NEAs, exponentially increasing potential damage. Thus, while comets are much less likely threats, they may also have much higher consequences and are typically much more challenging to mitigate. We are, therefore, motivated to understand how to address comets.

As an example, consider comet C/2013 A1 (Siding Spring). The comet was discovered in January 2013 and nearly struck Mars approximately 22 months later, in October of 2014. C/2013 A1 passed by Mars at a distance of approximately $140,496 \mathrm{~km}$ at closest approach, with a relative velocity of $56 \mathrm{~km} / \mathrm{s}$ (Farnocchia et al., 2016). This real-world event provides a sobering example of the sort of cometary Earth impact threat with which we could be confronted on short notice and underscores the need to include comet preparation in our planetary defense readiness activities.

\section{Key Questions to be Answered}

Below we list some unprioritized key questions to be answered through research and analysis efforts to advance planetary defense mission capabilities and readiness. We recommend the Planetary Defense portion of the forthcoming Planetary Science and Astrobiology Decadal Survey 2023-2032 include these key planetary defense research questions. The associated research and analysis efforts support completion of multiple actions in the NNPSAP, thus increasing our planetary defense preparedness. We anticipate NASA's Planetary Defense Coordination Office (PDCO) will play a key role in coordinating, within NASA and with other government agencies, over-arching plans for the research. Some of that coordination has already begun. We suggest that coordination continue with renewed focus, expanded scope, and increased resources suitable to addressing the research described here within the time horizon of the Decadal Survey 2023-2032. Finally, we also recommend that the investigation of less mature technology concepts for deflection/disruption should be supported at an appropriate level, per NNPSAP Action 3.4.

1. What envelopes of spacecraft mission performance parameters are appropriate to assume for credible, realistic assessments (particularly early assessments) of planetary defense campaign missions, including rapid-response reconnaissance and in-space mitigation (deflection/disruption) missions? What is the comprehensive set of relevant mission design and performance parameters, and what bounding values are appropriate for each, as functions of relevant scenario properties? [Helps address NNPSAP Action 3.6.]

2. Given current best knowledge of the anticipated total NEO population, what are the statistical descriptions, relative probabilities, and combinations of NEO orbital and physical properties that are most likely to pose future threat scenarios? Do those results identify gaps between our anticipated planetary defense mission campaign capabilities and the capabilities that might actually be required to deal with the more stressing potential cases? [Helps address NNPSAP Actions 3.1-3.7.]

3. Which NEO properties most strongly influence the outcomes of an impact on Earth, the application of a deflection or disruption system to an NEO, and other aspects of a planetary defense scenario? How should NEO properties be prioritized for characterization during a given type of planetary defense scenario, and what methods of 
characterization are required? How accurately do an NEO's properties need to be known in order to adequately assess the threat and plan effective mitigation efforts? [Helps address NNPSAP Actions 2.3, 2.5, 2.6, 3.1, and 3.3.]

4. What are the interior structures of NEOs like? The internal structure of an NEO has never been directly measured; however, interior structure (e.g., rubble pile vs. fractured shard vs. monolith) will influence the outcome of deflection, and particularly disruption, attempts. [Helps address NNPSAP Actions 2.3, 2.5, 2.6, 3.1 and 3.4-3.6.]

5. How much applied deflection "Delta-V" (change-in-velocity) can an NEO absorb without accidental fragmentation? When is the Delta-V too high? Will dividing the Delta-V into smaller applications via multiple spacecraft avoid accidental NEO fragmentation? The total number of spacecraft/launches required for a planetary defense mission campaign significantly drives cost, complexity, risk, and other key mission performance metrics. [Helps address NNPSAP Actions 2.3, 2.5, 2.6. and 3.4-3.6.]

6. What are the capabilities and limitations of high-speed spacecraft flyby missions for performing reconnaissance of potentially hazardous NEOs? Studies should assess how well high-speed spacecraft flybys can measure relevant NEO properties, as well as what technology development is needed to further enhance these reconnaissance capabilities. [Helps address NNPSAP Actions 3.1 and 3.3.]

7. How can the integration of fast probabilistic impact consequence models into mission design processes enable seamless, risk-informed mitigation mission design and optimization? What is a sufficient deflection impulse to confidently divert a threat with large physical property and orbit uncertainties? How does NEO disruption affect impact risk? Could intentional disruption of an NEO be desirable if minor damage is produced over a larger, less inhabited zone as opposed to major damage in a population center? [Supports NNPSAP Actions 2.3, 2.4, 2.5, 3.5, 3.6.]

8. To what extent can existing domestic and international launch vehicle infrastructures support planetary defense campaign missions, to include rapid-response reconnaissance and in-space mitigation (deflection/disruption) missions? How and to what extent can the world's existing launch infrastructure be made more responsive? Can new paradigms in launch vehicle procurement and retention, e.g., the "launch on need (LON)" system described herein, enable sufficiently responsive launch support for planetary defense missions? [Helps address NNPSAP Action 3.2.]

9. What is the set of plausible architectures for planetary defense campaign missions, to include rapid-response reconnaissance and in-space mitigation (deflection/disruption) missions? For example, to what extent should planetary defense spacecraft hardware be pre-fabricated versus built on demand, and to what extent should custom-built components be utilized versus commercial-off-the-shelf components? What are the capabilities, limitations, advantages, disadvantages, costs, and other performance parameters of the various identified architectures? How do the various possible architectures compare to one another? [Helps address NNPSAP Actions 3.1, 3.3, and 3.6.]

10. What are the ranges of mission performance requirements associated with the spectrum of plausible long-period/hyperbolic comet planetary defense scenarios? What are the gaps in current and foreseen capabilities for rapid-response reconnaissance and in-space mitigation (deflection/disruption) of such comets, and how might those gaps be closed? [Helps address NNPSAP Actions 3.1-3.6.] 


\section{References}

Barbee, B. W. (2020). "Near-Earth Object Characterization Priorities For Planetary Defense," Presentation to the $22^{\text {nd }}$ NASA SBAG Meeting, https://www.lpi.usra.edu/sbag/meetings/jan2020/presentations/Barbee_NEO_Characteriz ation_PD_SBAG_2020-01-16.pdf

Bhaskaran, S., and Kennedy, B. (2014). Closed Loop Terminal Guidance Navigation for a Kinetic Impactor Spacecraft. Acta Astronautica, 2014, vol 103, pp 322-332.

Caffrey, B. (2019). Rideshare/Multi-Manifest Payload Overview. Retrieved May 9, 2020, from https:/cubesats.gsfc.nasa.gov/symposiums/2019/presentations/Day2/CAFFREY.pdf

Dearborn, D., Syal, M., Barbee, B. G., Greenaugh, K., Howley, K., Leung, R., . . Zebenay, M. (2020). Options and uncertainties in planetary defense: Impulse-dependent response and the physical properties of asteroids. Acta Astronautica, 290-305.

Farnocchia, D., et al. (2016). High precision comet trajectory estimates: The Mars flyby of C/2013 A1(Siding Spring). Icarus, 266 (2016) 279-287. http://dx.doi.org/10.1016/j.icarus.2015.10.035.

Hernandez, S., Barbee, B., Bhaskaran, S., and Getzandanner, K. (2013). "Mission Opportunities for the Flight Validation of the Kinetic Impactor Concept for Asteroid Deflection," IAA Planetary Defense Conference, 2013, IAA-PDC13-04-09.

Housen, K., \& Holsapple, K. (2011). Ejecta from imapct craters. Icarus, 856-875.

IAA Planetary Defense Conference. (2019). Conference Summary and Recommendations. Washington, DC, USA: International Academy of Astronautics.

National Science \& Technology Council (NSTC). (2018). National Near-Earth Object Preparedness Strategy and Action Plan. Washington D.C.: Executive Office of the President of the United States.

Rumpf, C. M. (2016). Asteroid Impact Risk. Southampton, 125-126, UK: University of Southampton.

Rumpf, C. M., Mathias, D. L., Wheeler, L. F., Dotson, J. L., Barbee, B., Roa, J., . . Farnocchia, D. (2020). Deflection Driven Evolution of Asteroid Impact Risk Under Large Uncertainties. Acta Astronautica, accepted.

Sarli, B.V., and Y. Tsuda, Hayabusa 2 Extension Plan: Asteroid Selection and Trajectory Design. Acta Astronautica, 2017, vol 138, pp 225-232.

Silber, E. A., Boslough, M., Hocking, W. K., Gritsevich, M., \& Whitaker, R. W. (2018). Physics of Meteor Generated Shock Waves in the Earth's Atmosphere - A Review. Advances in Space Research, 489-532

Skylab Rescue. (2020, February 27). Retrieved May 9, 2020, from https://en.wikipedia.org/wiki/Skylab_Rescue

Soderman/NLSI Staff. (2013). The Chelyabinsk Impact of 02/15/13. Retrieved May 9, 2020, from https://sservi.nasa.gov/articles/the-chelyabinsk-impact-of-021513/

Stickle, A., et al. (2020). The Need For a Well-defined Modeling Pipeline For Planetary Defense. White paper submitted to The Planetary Science and Astrobiology Decadal Survey 20232032.

STS-3xx. (2020, May 27). Retrieved May 9, 2020, from https://en.wikipedia.org/wiki/STS-3xx

Wheeler, L.F., Mathias, D.L., Stokan, E., Brown, P.G., 2018. Atmospheric energy deposition modeling and inference for varied meteoroid structures. Icarus 315, 79-91. https://doi.org/10.1016/j.icarus.2018.06.014 